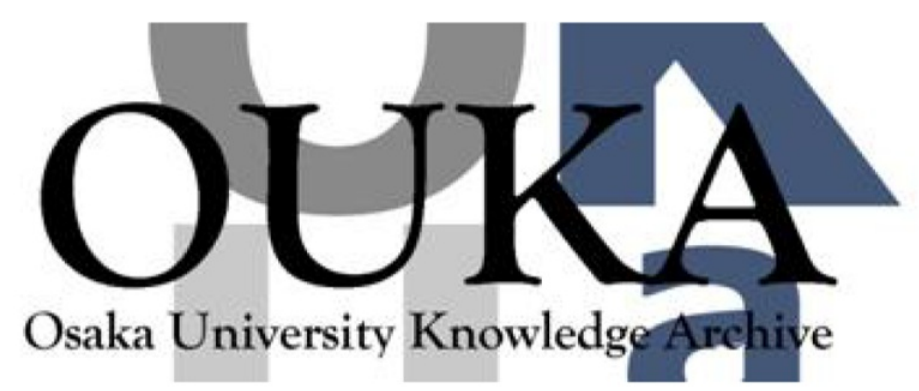

\begin{tabular}{|c|l|}
\hline Title & $\begin{array}{l}\text { Scalable spin amplification with a gain over a } \\
\text { hundred }\end{array}$ \\
\hline Author(s) & $\begin{array}{l}\text { Negoro, Makoto; Tateishi, Kenichiro; Kagawa, } \\
\text { Akinori et al. }\end{array}$ \\
\hline Citation & Physical Review Letters. 107(5) p. 050503 \\
\hline Issue Date & $2011-07-28$ \\
\hline oaire:version & VoR \\
\hline URL & https://hdl. handle. net/11094/77649 \\
\hline rights & $\begin{array}{l}\text { Copyright (2011) by the American Physical } \\
\text { Society }\end{array}$ \\
\hline Note & \\
\hline
\end{tabular}

Osaka University Knowledge Archive : OUKA

https://ir. Library. osaka-u. ac. jp/

Osaka University 


\title{
Scalable Spin Amplification with a Gain Over a Hundred
}

\author{
Makoto Negoro, Kenichiro Tateishi, Akinori Kagawa, and Masahiro Kitagawa \\ Graduate School of Engineering Science, Osaka University, Toyonaka, Osaka, 560-8531, Japan
}

(Received 25 February 2011; published 28 July 2011)

\begin{abstract}
We propose a scalable and practical implementation of spin amplification which does not require individual addressing nor a specially tailored spin network. We have demonstrated a gain of 140 in a solidstate nuclear spin system of which the spin polarization has been increased to 0.12 using dynamic nuclear polarization with photoexcited triplet electron spins. Spin amplification scalable to a higher gain opens the door to the single spin measurement for a readout of quantum computers as well as practical applications of nuclear magnetic resonance spectroscopy to infinitesimal samples which have been concealed by thermal noise.
\end{abstract}

DOI: 10.1103/PhysRevLett.107.050503

PACS numbers: 03.67.Lx, 76.60.- k, 76.70.Fz

A magnetic moment of a single nuclear spin is so small that its induction signal is buried under thermal noise. The minimum detectable number of perfectly polarized nuclear spins is still in the order of $10^{6}$ with a cryogenic inductive detection circuit. If information of a single nuclear spin is quantum-logically copied to a large number of spins, a spin component can be amplified and hence its signal may be detected with sufficient signal-to-noise ratio (SNR). This scheme is called spin amplification, which can be realized by the quantum circuit shown in Fig. 1(a) [1]. Although copy of an arbitrary, unknown state itself is prohibited by the no-cloning theorem [2], the information about whether the state is in $|0\rangle$ or $|1\rangle$ can be copied. Figure 1(a) can be used for a readout of quantum computers.

We propose other potential applications of spin amplification. To experimentally determine an arbitrary, unknown quantum state $|\psi\rangle$ of a spin, quantum state tomography is used, in which the state $|\psi\rangle$ is repeatedly prepared and measured [3]. If the number of repetitions is $N$ and the measurement is limited by the thermal noise of the detection apparatus, then the SNR is increased by a factor of $\sqrt{N}$. If the state is prepared and accumulated elsewhere to make $N$ identical copies of $|\psi\rangle$, as shown in Fig. 1(b), and measured at once, then the signal becomes $N$ times as large and the SNR is increased by a factor of $N$, which is nontrivial because it is much more efficient than $\sqrt{N}$ attained by the simple repetition. This scheme can also be regarded as amplification in a broader sense because it improves the SNR against the noise entering afterwards. The spin amplification [Fig. 1(b)] can be applied to quantum metrology which estimates a small unknown parameter $\phi$ contained in a quantum evolution $U$ from the initial state $|0\rangle$ to the final state $|\psi\rangle$ [4]. This procedure is regarded as spectroscopy as well when the parameter $\phi$ is given by an internal Hamiltonian of a spin system of interest. The minimum detectable value of $\phi$ is improved by a factor of $N$ as long as it is limited by the thermal noise of the detection apparatus.
In the $S-I^{m}$ spin system (a rarely existing $S$ spin and $m$ abundant $I$ spins), the absolute maximum gain of spin amplification shown in Figs. 1(a) and 1(b) is limited by $m$. To increase the maximum gain, $m$ must be increased. Figures 1(a) and 1(b) are, in principle, scalable, but they hardly scale up to larger $m$ in practice because they require individual addressing to all spins or a special spin network with tailored couplings such as a spin chain and a star topology [5-8]. Until now, a gain as low as four has been demonstrated with nuclear spins in molecules in solution $[8,9]$. Building a spin amplifier with a high gain, say, 100, seems as daunting as building a 100-qubit quantum computer.

In this Letter, we propose a new scalable implementation of spin amplification shown in Fig. 1(c), which utilizes spin diffusion [10] and magnetic-field cycling [11]. The total Hamiltonian of an $S-I^{m}$ nuclear spin system with a dipolar coupling network, naturally existing in a bulk solid, is given by (a)
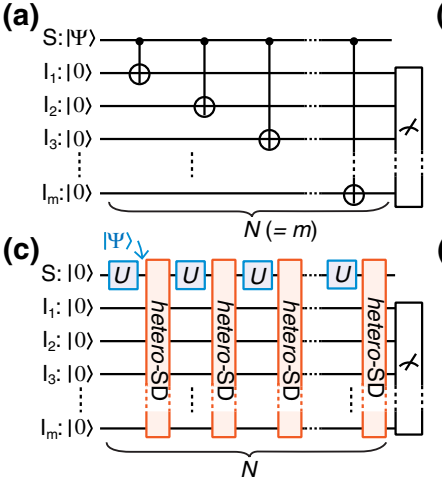

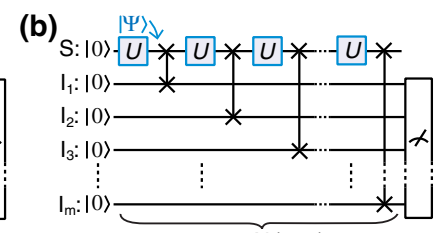

(d)

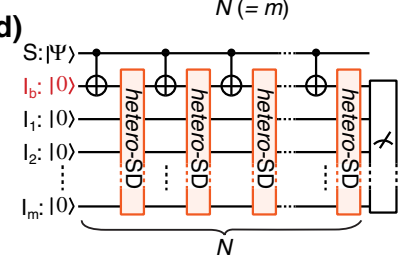

FIG. 1 (color online). (a) Spin amplification referred to by DiVincenzo [1]. (b) Spin amplification of the $S$ spin response signal using selective SWAP gates and (c) that using the heteroSD process. (d) Scalable version of (a). The $I$ spins are initialized to $|0\rangle$ for simplicity, although they do not have to be in practice. 


$$
\mathcal{H}=\omega_{S} S_{Z}+\omega_{I} \sum_{i} I_{i Z}+\sum_{i, j} \mathcal{H}_{i, j}+\sum_{i} \mathcal{H}_{i, S},
$$

where $\omega_{S}$ and $\omega_{I}$ are the resonance frequencies of the rare spin $S$ and the abundant spins $I . \mathcal{H}_{i, j}$ and $\mathcal{H}_{i, S}$ represent the homonuclear and the heteronuclear dipolar interactions, respectively. The dipolar interaction, $\mathcal{H}_{i, j}$, between the $i$ th spin $I_{i}$ and the $j$ th spin $I_{j}$ (or $S$ for the heteronuclear case, $\mathcal{H}_{i, S}$ ) is given by $\mathcal{H}_{i, j}=\mathcal{H}_{i, j}^{Z Z}+\mathcal{H}_{i, j}^{X Y}$, where $\mathcal{H}_{i, j}^{Z Z}=d_{i, j} I_{i Z} I_{j Z}$ (for the heteronuclear case, the dipolar coupling strength $d_{i, j}$ is replaced with $d_{i, S}$ ) generates mutually controlled rotations about the $z$ axis and $\mathcal{H}_{i, j}^{X Y}=$ $-d_{i, j}\left(I_{i X} I_{j X}+I_{i Y} I_{j Y}\right) / 2$ generates fractional power of $i$ SWAP operations. Both are always on between homonuclear spins regardless of the static magnetic field strength. Between heteronuclear spins, the former is always on and the latter is turned off in high static magnetic fields by the Zeeman energy difference being much larger than the dipolar interaction strength $\left(\left|\omega_{S}-\omega_{I}\right| \gg\left|d_{i, S}\right|\right)$. In low fields $\left(\left|\omega_{S}-\omega_{I}\right| \lesssim\left|d_{i, S}\right|\right)$, the latter is turned on. Under free time evolution of the total Hamiltonian in the high field, the $z$ components of only the $I$ spins are shuffled by simultaneous fractional powers of $i$ SWAP operations, and this behavior is known as spin diffusion [10]. In the low field, the $S$ spin is also involved in the spin diffusion among the $I$ spins via its coupling to at least one $I$ spin: we call it the heteronuclear-spin-involved spin diffusion (hetero-SD) process, where the total $z$ spin component is preserved. The hetero-SD process can be switched on and off by magneticfield cycling between the low and the high fields [11-13]. Therefore the quantum circuit of Fig. 1(c) can be easily realized by the simple repetition of synchronized field cycling and a rf pulse irradiation for the quantum operation $U$ to the $S$ spin alone. The process requires only a reasonably dense network of dipolar-coupled spins without requiring the tailored coupling strength $\left(d_{i, j}\right)$, and, therefore, is quite general, robust, and practical in real samples which may have some irregularities or randomness.

By exciting the $z$ component of the $S$ spin with a rf pulse as $U$ and subsequently switching on the hetero-SD process for a sufficiently long duration, as shown in Fig. 1(c), the excited $z$ component of the $S$ spin is diffused in the reservoir of the abundant $I$ spins. By repeating the sequence, the excitations are accumulated in the abundant $I$ spins. The information about whether the $S$ spin is inverted by $U$ is converted into the decrease in the $z$ component of the total magnetization of $m$ abundant $I$ spins, which is proportional to the $I$ spin polarization. Assuming that the polarizations of the $S$ spin and the $I$ spins are uniformly mixed in the hetero-SD process, the $I$ spin polarization after the $N$ th repetition of the $U=$ NOT and the hetero-SD process decreases to $\epsilon_{0}[(m-1) /(m+1)]^{N}$ from the initial polarization $\epsilon_{0}$ of the $S$ and the $I$ spins. Compared with the difference between the directly observed signal of the $S$ spin of polarization $\epsilon_{0}$ and that of the inverted $S$ spin, the

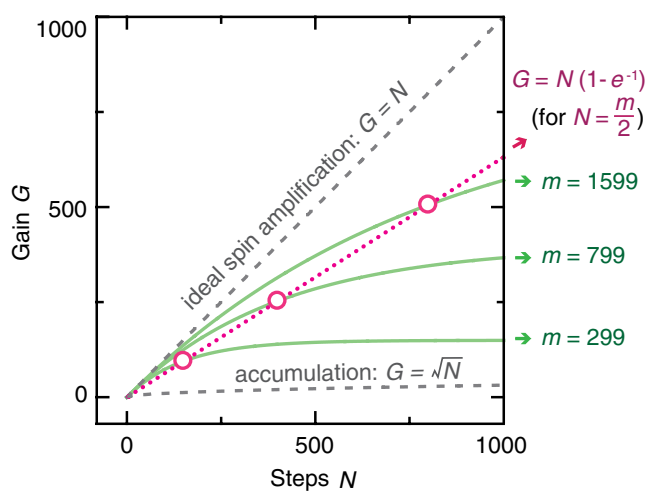

FIG. 2 (color online). The top dashed line shows the gain $G$ of the SNR of ideal spin amplification [Figs. 1(a) and 1(b)] with respect to the number of SWAP gates $N$. The solid lines show the expected gain [Eq. (2)] of the proposed one [Fig. 1(c)] with respect to the number of the hetero-SD process gates $N$ for different system sizes. The bottom dashed line shows the gain of the direct simple repetitive detection with respect to the number of repetitions $N$.

expected gain of the signal obtained from the polarization difference of $m$ abundant $I$ spins at the $N$ th step of spin amplification with and without $U$ is given by

$$
\begin{aligned}
G & =\frac{m\left\{\epsilon_{0}-\epsilon_{0}[(m-1) /(m+1)]^{N}\right\}}{\epsilon_{0}-\left(-\epsilon_{0}\right)} \\
& =\frac{m}{2}\left[1-\left(\frac{m-1}{m+1}\right)^{N}\right] .
\end{aligned}
$$

In an $S-I^{m}$ spin system, the gain of the spin amplification using the hetero-SD process increases with $N$ but is saturated to $m / 2$ for $N \gg m / 2$, as shown in Fig. 2. The proposed implementation using the hetero-SD process gives better SNR improvement with respect to $N$ than the simple $N$ repetitive detection. When $m$ and $N$ are increased simultaneously while keeping $N=m / 2$, the amplification gain scales up linearly as $m\left(1-e^{-1}\right) / 2$ (the dotted line) with respect to $m$ ( $\gg 1)$.

The quantum circuit of Fig. 1(d) is a scalable version of Fig. 1(a) and could be used for a readout of quantum computers [14]. While there is a functional difference between Figs. 1(d) and 1(c), the most difficult part, the scalability of implementation to achieve a higher gain, is common in Figs. 1(d) and 1(c). In Fig. 1(d), the information about whether the state of a rare spin $S$ is $|0\rangle$ or $|1\rangle$ is nondestructively copied to a buffer spin $I_{b}$ by the CNOT gate and accumulated in abundant $I$ spins with hetero-SD processes. The field cycling between a high field $\left(\mid \omega_{I}-\right.$ $\omega_{I b} \mid \gg d_{I, I b}$ and $\left.\left|\omega_{S}-\omega_{I b}\right| \gg d_{S, I b}\right)$ and a low field $\left(\left|\omega_{I}-\omega_{I b}\right| \sim d_{I, I b}\right.$ and $\left.\left|\omega_{S}-\omega_{I b}\right| \gg d_{S, I b}\right)$ enables the hetero-SD process in Fig. 1(d). Our implementations, both Figs. 1(c) and 1(d), using the field cycling do not require rf irradiation to the abundant $I$ spins, of which a subtle imperfection causes the large degradation of the spin amplification gain. 

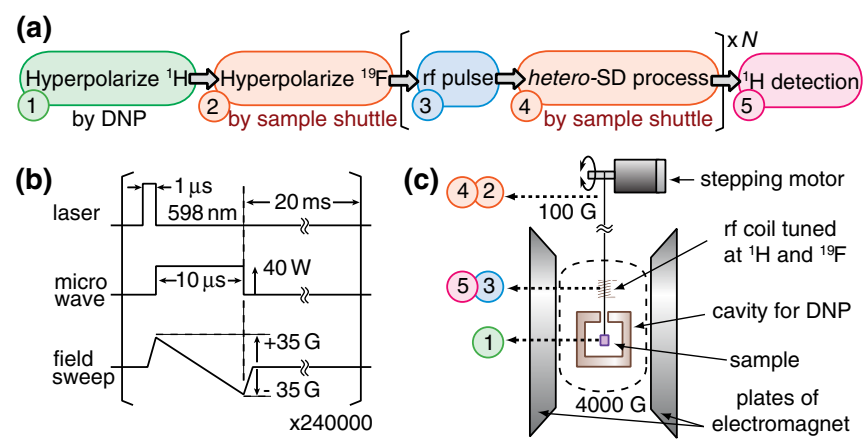

FIG. 3 (color online). (a) An experimental procedure of spin amplification using the hetero-SD process [15]. (b) A DNP sequence with integrated solid effect [16]. (c) A schematic diagram of an experimental setup where the sample position at each stage is indicated.

In this work, we have performed experiments to amplify the response of a rare spin with respect to a rf pulse and demonstrated a spectroscopic application in an $S-I^{799}$ spin system in a bulk solid with the quantum circuit shown in Fig. 1(c). We used a sample of a single crystal of naphthalene doubly doped with $\sim 0.005 \mathrm{~mol} \%$ pentacene and $\sim 1 \mathrm{~mol} \%$ 2-fluoronaphthalene. If the sample is uniform, it is approximately regarded as the ensemble of $S-I^{799}$ spin systems because a ${ }^{19} \mathrm{~F}$ spin is surrounded by $799{ }^{1} \mathrm{H}$ spins on average. The experimental procedure is shown in Fig. 3(a) [15]. In the first stage of every experiment, we enhanced the ${ }^{1} \mathrm{H}$ spin polarization to $\sim 0.12$ at $233 \mathrm{~K}$ by dynamic nuclear polarization (DNP) with photoexcited triplet electron spins of pentacene molecules [16-19]; the sequence of the photoexcitation and the integrated solid effect [16] are shown in Fig. 3(b).

The key stage in this experiment, the hetero-SD process, was realized by field cycling $(4000 \mathrm{G} \rightarrow 100$ $\mathrm{G} \rightarrow 4000 \mathrm{G}$ ) using sample shuttling, as shown in Fig. 3(c). In $100 \mathrm{G}$, the resonance frequency difference between the ${ }^{1} \mathrm{H}$ and the ${ }^{19} \mathrm{~F}$ spins is of the same order as the strength of the nearest neighbor ${ }^{1} \mathrm{H}-{ }^{19} \mathrm{~F}$ dipolar coupling. After repeating a rf pulse to ${ }^{19} \mathrm{~F}$ spins as the quantum operation $U$ and the sample shuttling $N$ times, we observed the ${ }^{1} \mathrm{H}$ magnetization.

We investigated the behavior of the ${ }^{1} \mathrm{H}$ spin polarization with respect to the number of steps $N$ of spin amplification [Fig. 4(a)]. In the case of $U=I$ with no rf pulse, the ${ }^{1} \mathrm{H}$ spin polarization decayed with $\eta$ of $\sim 99.91 \%$ per cycle, which gave close agreement with the decay rate calculated from $T_{1}$ measured at each sample position. In the case of an on-resonance $\pi$ pulse (i.e., $U=\mathrm{NOT}$ ), the result was close to the theoretical decay $\epsilon_{0}\{(m-1) /(m+1)\}^{N} \eta^{N}$ (the dashed line) calculated from the decreased polarization in Eq. (2) and the decay $\eta$. The $S$ spin response to $U$ is converted into the difference between the $I$ spin polarization with and without $U$. The polarization difference was amplified 37 times for $N=40$ and 136 times for $N=200$, compared with that for $N=1$. The amplification gain

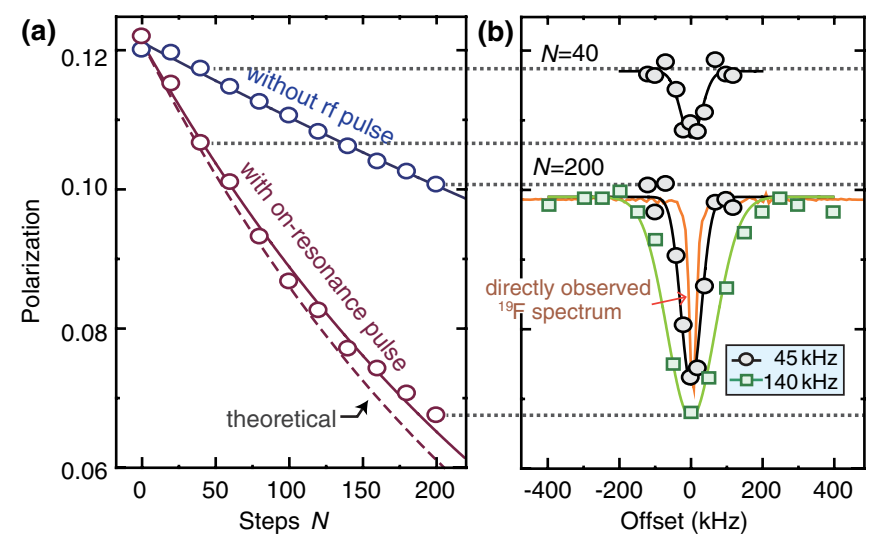

FIG. 4 (color online). (a) The behaviors of the ${ }^{1} \mathrm{H}$ spin polarization with respect to the number of steps $N$ of spin amplification without rf pulse $(U=I)$ and with on-resonance $\pi$ pulses $(U=$ NOT $)$. The dashed line is the theoretical decay. (b) The circles show the spin-amplified frequency response spectra obtained for $N=40$ and 200. They were obtained with the rf pulse with a peak amplitude of $45 \mathrm{kHz}$ (the pulse length is $\sim 3$ times as long as that of $140 \mathrm{kHz}$ ). The squares show the spin-amplified frequency response spectra obtained for $N=200$ with the $\mathrm{rf}$ pulse with a peak amplitude of $140 \mathrm{kHz}$. Directly detected ${ }^{19} \mathrm{~F}$ spectrum vertically magnified is also shown for comparison.

anticipated from Eq. (2) is 38 for $N=40$ and 157 for $N=200$. By taking into account the frequencies for ${ }^{19} \mathrm{~F}$ and ${ }^{1} \mathrm{H}$ spins, the signal obtained from the polarization difference of the ${ }^{1} \mathrm{H}$ spins with and without $U=$ NOT was estimated to be $\sim 140$ times as large as that of the ${ }^{19} \mathrm{~F}$ spin with the polarization 0.11 . It is noteworthy that the 140-time amplified signal is more than 10 times as large as the signal of the perfectly polarized ${ }^{19} \mathrm{~F}$ spins. Furthermore, a factor of 140 spin amplification in conjunction with the modern magnetic resonance force microscopy [20] technology, which is expected to detect 100 nuclear spins in the near future [21], may open the door to the detection of a faint signal from a single nuclear spin.

Amplified responses to the rf pulses with various carrier frequencies for $N=200$ give the excitation spectrum of the ${ }^{19} \mathrm{~F}$ spin, as represented by the squares in Fig. 4(b). The spin-amplified response spectra with the weaker rf pulse (the circles) were narrower than that but still broader than the directly observed ${ }^{19} \mathrm{~F}$ NMR spectrum. The pulse intensity should be lowered in order to avoid power broadening. The depth of the spectral response was increased with $N$.

All spin-amplified ${ }^{1} \mathrm{H}$ NMR signals were reduced by a factor of 100 on purpose to avoid saturation of the detection circuit [15]. With the reduction, the directly observed ${ }^{19} \mathrm{~F}$ NMR signal was completely concealed with the thermal noise. The ${ }^{19} \mathrm{~F}$ signal observed without the reduction is shown in Fig. 4(b). If the sample size is 100 times as small, the ${ }^{19} \mathrm{~F}$ signal cannot be detected even without the reduction. On the other hand, the spin-amplified ${ }^{1} \mathrm{H}$ signals of the small sample can be detected with the approximately 
same SNR as the present result without the reduction. Spin amplification will have a distinct advantage over the simple repetitive detection in a smaller or a more diluted sample. If we use a sample with a lower 2-fluoronaphthalene concentration, we could get a higher gain by increasing $N$ in the same manner. The maximum achievable gain is inversely proportional to the concentration. It has been reported that $T_{1}$ of the ${ }^{1} \mathrm{H}$ spins in naphthalene doped with $\sim 0.01 \mathrm{~mol} \%$ pentacene in $\sim 7 \mathrm{G}$ at $77 \mathrm{~K}$ is $\sim 166 \mathrm{~min}$ [18], and the result implies that $T_{1}$ of a sample with a lower 2-fluoronaphthalene concentration at a lower temperature is longer. In addition, there is room for speeding up field cycling [11,22], and hence earning the number of steps $N$ well before the ${ }^{1} \mathrm{H}$ polarization vanishes by $T_{1}$ relaxation. Scalable spin amplification will enable practical applications of NMR spectroscopy to rarely existing nuclear spins in infinitesimal bulk samples which have been concealed by thermal noise. For higher sensitivity and spectral resolution, the quantum operation $U$ and the NMR detection should be performed in a higher magnetic field, which is realized by the field-cycling system described in [23].

In the proposed implementation using field cycling for switching the hetero-SD process, the nuclear species are modestly restricted to those with the resonant frequency close to that of the abundant ${ }^{1} \mathrm{H}$ spins. For other nuclear species to be involved in spin diffusion process, the double resonance irradiation satisfying the Hartmann-Hahn condition [24], as in the cross polarization experiment [25], may be used, although it is subject to the rf pulse imperfection and the much faster decay with $T_{1 \rho}$. State-of-the-art high fidelity pulse engineering [26,27] may be required to implement high-gain spin amplification.

The demonstrated spin amplification [Fig. 1(c)] can be straightforwardly modified to Fig. 1(d). It can be realized, for example, by doping $2-{ }^{13} \mathrm{C}$ 2-fluoronaphthalene, in which the ${ }^{13} \mathrm{C},{ }^{19} \mathrm{~F}$, and ${ }^{1} \mathrm{H}$ spins play the roles of the $S$, the $I_{b}$, and the $I$ spins, respectively. Recently, optically detected magnetic resonance $[28,29]$ has accomplished single-event state readout of a single nuclear spin [30], which is attracting attention in quantum information science. The breakthrough of the gain shown in this Letter is an important step for state readout of a single nuclear spin in bulk solids with more versatile detection such as magnetic resonance force microscopy and the inductive detection.

In this Letter, we have proposed a scalable and practical implementation of spin amplification and successfully demonstrated a gain as large as 140. A faint magnetization of a rare spin has been quantum-logically transferred beyond the molecular boundary to abundant spins in the bulk solid sample through spin diffusion process which does not require individual addressing nor a specially tailored spin network. The amplified signal obtained from the abundant spins is more than 10 times as large as that of the completely polarized rare spins. A spectroscopic application has been demonstrated by 140-time amplified frequency response spectra of the rare spin. It will enable practical applications of NMR spectroscopy to infinitesimal samples which have been concealed by thermal noise. A higher gain is possible in the sample with a lower concentration of rare spins. Spin amplification scalable to a higher gain opens the door to a single spin measurement and a readout of spin-based quantum computers.

We thank K. Takeda for fruitful discussions. This work was supported by the CREST program of JST, MEXT Grant-in-Aid for Scientific Research on Innovative Areas 21102004, the Funding Program for World-Leading Innovative R\&D on Science and Technology (FIRST), and the Global-COE program of Osaka University.

[1] D. P. DiVincenzo, Fortschr. Phys. 48, 771 (2000).

[2] W. K. Wooters and W. H. Zurek, Nature (London) 299, 802 (1982).

[3] M. A. Nielsen and I.L. Chuang, Quantum Computation and Quantum Information (Cambridge University Press, Cambridge, England, 2000).

[4] N. F. Ramsey, Molecular Beams (Oxford University Press, London, 1956).

[5] C. A. Peŕez-Delgado, M. Mosca, P. Cappellaro, and D. G. Cory, Phys. Rev. Lett. 97, 100501 (2006).

[6] A. Kay, Phys. Rev. Lett. 98, 010501 (2007).

[7] J. A. Jones et al., Science 324, 1166 (2009).

[8] J.-S. Lee, T. Adams, and A. K. Khitrin, New J. Phys. 9, 83 (2007).

[9] P. Cappellaro et al., Phys. Rev. Lett. 94, 020502 (2005).

[10] N. Bloembergen, Physica (Amsterdam) 15, 386 (1949).

[11] F. Noack, Prog. Nucl. Magn. Reson. Spectrosc. 18, 171 (1986).

[12] K. L. Ivanov, A. V. Yukovskaya, and H.-M. Vieth, J. Chem. Phys. 128, 154701 (2008).

[13] U. Bommerich et al., Phys. Chem. Chem. Phys. 12, 10309 (2010).

[14] Figure 1(d) is similar to the last scheme in Ref. [9] but is simpler than that. The latter relies on multiple pulse sequences to establish entanglement while the former does not require any rf irradiation to the abundant $I$ spins.

[15] See Supplemental Material at http://link.aps.org/ supplemental/10.1103/PhysRevLett.107.050503 for experimental details.

[16] A. Henstra, T.-S. Lin, J. Schmidt, and W. Th. Wenckebach, Chem. Phys. Lett. 165, 6 (1990).

[17] D. Stehlik and H.-M. Vieth, in Pulsed Magnetic Resonance NMR, ESR and Optics, edited by D.M.S. Bagguley (Oxford University Press, New York, 1992), pp. 446-477.

[18] M. Iinuma et al., Phys. Rev. Lett. 84, 171 (2000).

[19] K. Takeda, Triplet State Dynamic Nuclear Polarization (VDM Verlag, Saarbrücken, 2009).

[20] D. Rugar, R. Budakian, H. J. Mamin, and B. W. Chui, Nature (London) 430, 329 (2004).

[21] M. Poggio and C. L. Degen, Nanotechnology 21, 342001 (2010). 
[22] A. Krahn et al., Phys. Chem. Chem. Phys. 12, 5830 (2010).

[23] A. Kagawa, M. Negoro, K. Takeda, and M. Kitagawa, Rev. Sci. Instrum. 80, 044705 (2009).

[24] S. R. Hartmann and E. L. Hahn, Phys. Rev. 128, 2042 (1962).

[25] A. Pines, M. G. Gibby, and J. S. Waugh, J. Chem. Phys. 59, 569 (1973).
[26] N. Khaneja et al., J. Magn. Reson. 172, 296 (2005).

[27] C. A. Ryan, O. Moussa, J. Baugh, and R. Laflamme, Phys. Rev. Lett. 100, 140501 (2008).

[28] J. Kohler et al., Nature (London) 363, 242 (1993).

[29] J. Wrachtrup et al., Nature (London) 363, 244 (1993).

[30] P. Neumann et al., Science 329, 542 (2010). 